\title{
Reanalyzing the 1900-1920 Sleeping Sickness Epidemic in Uganda
}

\author{
E.M. Fèvre, ${ }^{*}$ P.G. Coleman, $\dagger$ S.C. Welburn, ${ }^{*}$ and I. Maudlin*
}

Sleeping sickness has long been a major public health problem in Uganda. From 1900 to 1920, more than 250,000 people died in an epidemic that affected the southern part of the country, particularly the Busoga region. The epidemic has traditionally been ascribed to Trypanosoma brucei gambiense, a parasite now confined to central and western Africa. The Busoga region still reports sleeping sickness, although it is caused by T.b. rhodesiense, commonly believed to have spread to Uganda from Zambia in the 1940s. Our analysis of clinical data recorded in the early 1900s shows that the clinical course of sleeping sickness cases during the 1900-1920 epidemic in Uganda was markedly different from T.b. gambiense cases, but similar to T.b. rhodesiense. These findings suggest that T.b. rhodesiense was present in Uganda and contributed to the epidemic. The historic context is reassessed in the light of these data.

$\mathrm{U}$ ganda is affected by Gambian sleeping sickness, which is caused by infection with Trypanosoma brucei gambiense, and Rhodesian sleeping sickness, which is caused by T.b. rhodesiense. T.b. rhodesiense occurs in the eastern part of the country, whereas T.b. gambiense occurs in the northwestern part of the country. From 1900 to 1920, the Busoga region of Uganda experienced a large-scale epidemic of the disease, during which an estimated 250,000 people (1), a third of the population of the region, died (Figure 1). It is believed that the species of parasite responsible for this first documented epidemic in Uganda was T.b. gambiense and that T.b. rhodesiense was introduced there in the 1940s when another, smaller epidemic was identified in the same region. However, this idea has been the subject of some debate $(2,3)$.

The first published description of sleeping sickness cases in the 1900-1920 epidemic was made by A.R. and J.H. Cook in their Church Missionary Society (CMS)

*University of Edinburgh, Roslin, Midlothian, United Kingdom and †London School of Hygiene and Tropical Medicine, London, United Kingdom
Hospital at Mengo on February 11, 1901 (4,5). The extent of the epidemic became clear as the number of casepatients seen at that hospital increased and as the disease was identified around the northern shore of Lake Victoria $(6,7)$. The study of sleeping sickness at the time focused on discovering the causative agent; being newly recognized in Uganda, the disease had not been previously described in eastern Africa. The discovery of trypanosomes as a disease agent and their mode of transmission was relatively recent. Bruce (8) described transmission of cattle trypanosomes by tsetse flies in Zululand in 1895; the causative agent of "Trypanosoma fever" in the Gambia had been indicated in 1902 by Forde (9) and described by Dutton (10) as Trypanosoma gambiense (now classified as T.b. gambiense). At the time of the Ugandan outbreak, Manson (11) believed that the disease was linked to Filaria perstans (now known as Mansonella perstans), a blood-dwelling nematode of no clinical importance. Early efforts in Ugandan patients infected with sleeping sickness focused on detection of F. perstans. T.b. rhodesiense was described for the first time (in present day Zambia) in 1910 (12), and T.b. rhodesiense was confirmed in Uganda during the next major epidemic 30 years later (13).
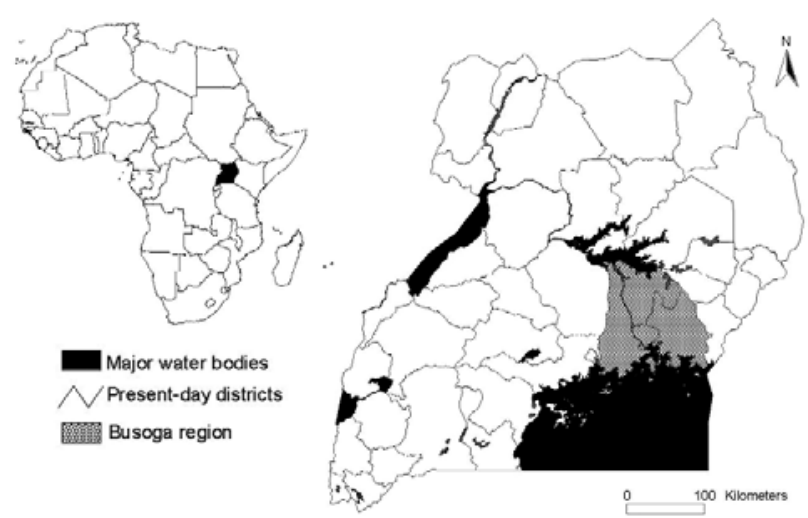

Figure 1. Map of Uganda showing its location in East Africa (inset) and the location of the Busoga region where approximately 250,000 people died from 1900 to 1920 . 


\section{A New Disease?}

It is not known how long sleeping sickness may have existed in Uganda before 1900. Most of the pioneering scientists assumed it was a new problem to the region because they found no evidence that it had occurred there before, and they assumed that the disease always occurred as an epidemic. However, Christy (7) noted that sleeping sickness had probably been present long before it was first documented and that it probably originated in Busoga, the core of the present day T.b. rhodesiense focus. Similarly, on reviewing the available evidence, Duke (14) states that "some form of human trypanosomiasis" had occurred around the Ugandan shores of Lake Victoria prior to the epidemic.

Confusion over the existence of sleeping sickness in this area was intertwined with the available knowledge of the causative organism. We have already seen that T.b. gambiense was the only human-infective trypanosome to have been described at the time. Furthermore, sleeping sickness was recognized in many parts of central Africa (15). It had not been documented previously in eastern Africa, and the assumption was that the Ugandan disease was an extension of the epidemic raging westward in the (present day) Democratic Republic of Congo.

When the epidemic began in Uganda, Castellani (16) noticed two groups of distinct clinical symptoms among the patients. The infection in the first group he called Trypanosoma fever, as is was similar to the disease seen in the Gambia and ascribed to T. gambiense $(10,17)$. The second infection he called sleeping sickness, and tentatively called the trypanosome that he found in those cases $T$. ugandense (16). The distinction was essentially clinical; what he called sleeping sickness was a much more virulent infection than the Trypanosoma fever caused by T. gambiense. Bruce et al. (18) described two cases of a disease "not unlike Trypanosoma fever" in two patients who had recently come to Uganda (whom he termed Nubians). The patients, a policeman and a prisoner, had arrived from the present day Sudan, where T.b. gambiense occurs today. Bruce et al. (18) later insisted that the less acute "Trypanosoma fever" symptoms were simply the first stage of full-blown sleeping sickness caused by T. gambiense.

If the sleeping sickness had been due to T.b. gambiense, it would suggest that the parasite was imported from the west as part of large-scale human population movements that occurred at the time (15). Only Köerner et al. (2) have questioned the identity of the parasite responsible for the first Ugandan epidemic. They argue that as T.b. rhodesiense has occurred in stable endemic foci that can expand and cause epidemics, T.b. rhodesiense was probably present in Busoga long before 1901. This is an attractive argument, as the wholesale replacement of one parasite species by another (T.b. gambiense by T.b. rhodesiense) in a region seems unlikely, and such a replacement has not been recorded in any other sleeping sickness focus. In addition, T.b. rhodesiense is primarily a zoonotic parasite in which human-to-human transmission is thought to occur rarely, and human movements $(15,19)$ in isolation from movements of the zoonotic reservoir (20) may not be sufficient to account for its introduction.

\section{Hypothesis}

Here we test the hypothesis proposed by Köerner et al. (2) that the parasite responsible for sleeping sickness in the Busoga and surrounding regions of Uganda from 1900 was T.b. rhodesiense. This organism causes an acute disease, resulting in death after a period of 3-12 months $(21,22)$, while T.b. gambiense causes a chronic infection, with which a person may go about daily activities for many months or years $(21,23)$, despite occasional and often mild symptoms. Our analysis, therefore, is based on comparison of the survival time of sleeping sickness patients estimated from clinical notes recorded during the Ugandan epidemic, with survival times of known $T$. $b$. rhodesiense patients from the current disease focus in southeast Uganda, and $T$. b. gambiense patients in western and central Africa.

\section{Methods}

\section{Archives}

The Mengo Hospital archives (CMS Mission Hospital at Mengo), which include original patient case notes made by the Cook brothers, are held in the archives section of the Mulago Hospital at the Makerere Medical School in Kampala, Uganda. The first sleeping sickness patient recognized in Mengo was admitted on February 11, 1901 (Figure 2). The geographic distribution of the cases seen at this hospital extended across a wide area, although most of the patients came from the close vicinity. Many patients reporting in the latter years of the epidemic were referred to the specialist hospital run by the Royal Society Sleeping Sickness Commission in Entebbe (set up as part of the Commission's study on the disease), or later to the sleeping sickness isolation camps on islands in Lake Victoria. Some details of the treatments prescribed and numbers of cases seen in these camps are available $(24,25)$. At this time in the development of therapy for sleeping sickness, no effective drugs were available for the disease, so death of the patients was due to sleeping sickness and, rarely, treatment side-effects; the data included here show the true clinical course of the disease in untreated patients.

\section{Data Collection}

For each patient treated at the Mengo Hospital with diagnosed sleeping sickness, full details as they appeared in the archives were entered into a database, which was 


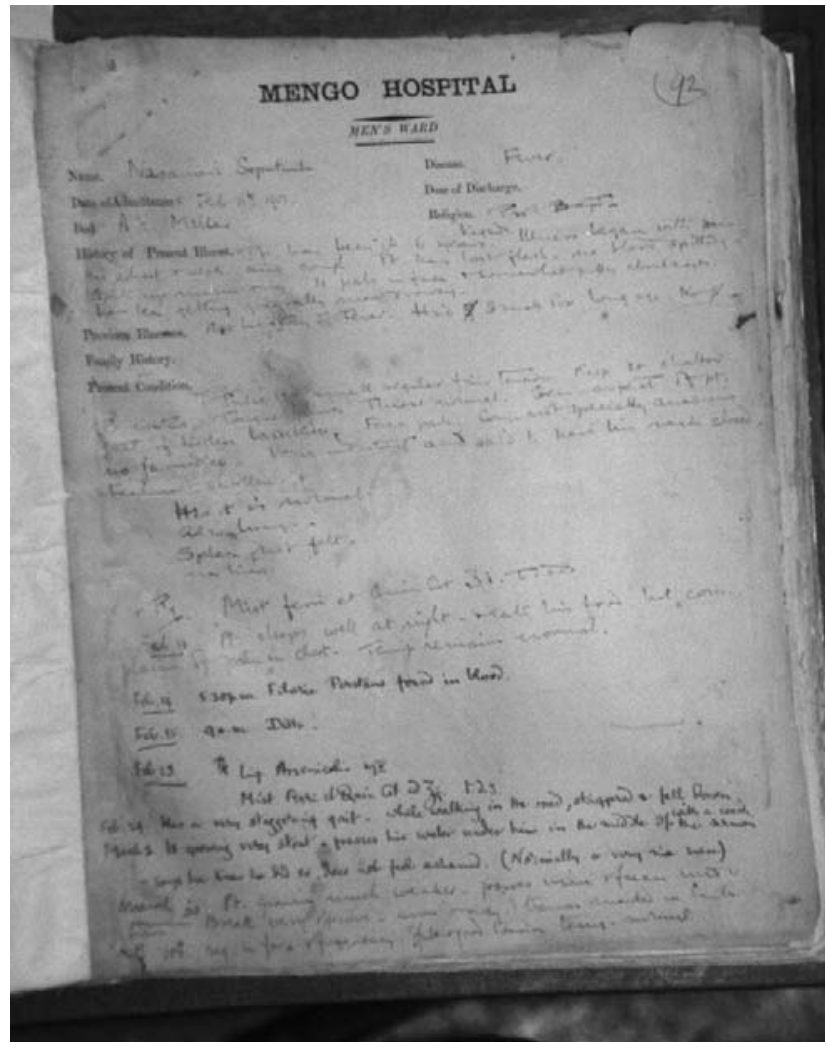

Figure 2. Case notes of the first recorded sleeping sickness patient in the Mengo Hospital case records.

used for later analyses. This electronic database has been made available to the archivists in Mengo. Full details were entered for patients seen through 1910; beyond this time, many patients were turned away. In addition, the authorities were managing to bring the epidemic under control, and the number of cases was diminishing. Additional data were also extracted from the Reports of the Royal Society Sleeping Sickness Commission $(6,18)$.

\section{T.b. rhodesiense Comparison}

Odiit et al. (22) published data on duration of symptoms in 30 patients who died of sleeping sickness within a week of seeking treatment for T.b. rhodesiense infection at the Livestock Research Institute sleeping sickness hospital in Tororo, Uganda, between 1988 and 1990.

\section{T.b. gambiense Comparison}

Adams et al. (26) conducted postmortem examinations on 16 patients with fatal T.b. gambiense infections and state that few fatal cases are documented and that duration of illness before death is "rarely established"; this accounts for the scarcity of time-to-death data in contemporary literature. One source of T.b. gambiense clinical data (27) presents important details of the clinical course of the disease to death but could not be used in this analysis as no estimates were made of the length of illness before treatment or death. However, Yorke (28) does present a summary of untreated cases between 1908 and1919 in various countries in West Africa. These countries include the French Congo (present-day Republic of Congo), the Gambia, and the Belgian Congo (present-day Democratic Republic of the Congo). Full details for each patient are not provided, and the survival times for the different groups of patients were taken as the average of the range given (e.g., five patients who were followed-up after 2-3 years were each given a survival time of 2.5 years). These data were supplemented by several more recently published case histories for T.b. gambiense patients who were treated in the United States and Europe after various periods of travel in Africa (23,29-33); the date of the last visit to Africa before diagnosis was taken as the infection date for these patients, and survival time was taken to the point of first treatment or death. Cases of congenital sleeping sickness from this literature were excluded.

\section{Statistical Analyses}

A survival analysis (34) was conducted to compare the time from onset of symptoms to death of the Ugandan patients in the Mengo archives and Sleeping Sickness Commission Reports $(6,18)$ from 1901 to 1910, and known T.b. rhodesiense (22) and T.b. gambiense patients $(23,28-33)$. The criteria for including a patient from the Mengo archive dataset were that the case had been recorded as a sleeping sickness death following inpatient stay in the hospital, that the length of the hospital stay was recorded, and that the length of time of illness before admission had been recorded in the clinical notes. That is, an estimate of the total time of the clinical course of the illness, from onset of symptoms to death, was available. The same criteria were applied to the clinical notes appearing in the Sleeping Sickness Commission Reports $(6,18)$. The Kaplan-Meier survival analyses were conducted in $S+2000$ (MathSoft, Inc., Cambridge, MA) and the survival curves were compared using the log-rank (Mantel-Cox) test.

\section{Results}

\section{Descriptive Statistics}

From 1901 to 1910, a total of 11,767 case-patients were recorded in the Mengo inpatient records. This figure excludes all patients admitted in 1902 because all the records from that year were destroyed in a fire. Just over $1 \%$ of these (204 cases) were sleeping sickness cases. The outcome of admission was biased in favor of discharge (160 cases). Five patients were referred to the Royal Society sleeping sickness hospital, and 24 deaths were recorded. Of these 24 deaths, 11 (Table 1) untreated 
Table 1. Time sick pre-admission and total time to death for 11 patients admitted to Mengo Hospital, $1901-1910$

\begin{tabular}{|c|c|c|c|c|c|c|c|}
\hline No. & Sex & Approx. age (y) & $\begin{array}{c}\text { Months sick } \\
\text { pre-admission }\end{array}$ & Admission date & Date of death & Time in hospital (mo) & Time sick predeath (mo) \\
\hline 2 & $\mathrm{~F}$ & 11 & 3 & Feb 13, 1901 & Mar 8, 1901 & 0.77 & 3.77 \\
\hline 3 & M & 30 & 1 & Apr 20, 1901 & May 8, 1901 & 0.60 & 1.60 \\
\hline 4 & $\mathrm{~F}$ & 10 & 6 & Apr 25, 1901 & May 8, 1901 & 0.43 & 6.43 \\
\hline 13 & M & - & 3 & Jul 29, 1901 & Aug 28, 1901 & 1.00 & 4.00 \\
\hline 18 & M & - & 3 & Sep 16, 1901 & Oct 19, 1901 & 1.10 & 4.10 \\
\hline 26 & M & 18 & 0.03 & Jun 28, 1903 & Jul 4, 1903 & 0.20 & 0.23 \\
\hline 133 & M & 20 & 0.20 & Jun 23, 1907 & Jul 8, 1907 & 0.50 & 0.70 \\
\hline 136 & M & 40 & 24 & Jul 3, 1907 & Jul 10, 1907 & 0.23 & 24.23 \\
\hline 171 & $\mathrm{~F}$ & 40 & 1 & Apr 24, 1909 & May 4, 1909 & 0.33 & 1.33 \\
\hline 181 & M & 20 & 5 & Mar 1, 1910 & Mar 12, 1910 & 0.37 & 5.37 \\
\hline 189 & M & 45 & 1 & Jun 9, 1910 & Jun 24, 1910 & 0.50 & 1.50 \\
\hline
\end{tabular}

patients had complete records of date of admission and death and of the duration of symptoms before admission. Sixteen cases from the Sleeping Sickness Commission Reports were included with these (Table 2). All 30 cases presented by Odiit et al. (22) were included, and 88 untreated, diagnosed case-patients were extracted from Yorke (28). Of these, 54 had died and 34 were still alive on follow-up (accounted for by censoring in the survival analysis). Eight contemporary T.b. gambiense patients were included $(23,29-33)$, all of whom survived to treatment.

\section{Survival Curves}

The Kaplan-Meier Survivorship curves resulting from the analysis of these data are shown in Figure 3. The median survival times were 2 months, 4 months, and 36 months for the Tororo 1988-1990, Mengo Hospital Plus Sleeping Sickness Commission reports 1901-1910, and western African datasets, respectively.

\section{Log-Rank Test}

The log-rank test showed no significant difference between the Ugandan 1901-1910 and 1988-1990 survival rates $\left(\chi^{2}=1.7 ; d f=1, \mathrm{p}=0.12\right)$. Therefore, the clinical course from onset of symptoms to death in this sample of patients from the 1901-1910 epidemic in southern Uganda was not significantly different to that of patients in Tororo with T.b. rhodesiense infections from 1988 to 1990. The Ugandan 1901-1910 and West African survival rates were significantly different $\left(\chi^{2}=184 ; d f=1, \mathrm{p}<0.001\right)$ and the Ugandan 1988-1990 and West African survival rates were also significantly different $\left(\chi^{2}=175 ; d f=1, \mathrm{p}<0.001\right)$. The clinical course of the disease during the Ugandan 1901-1910 and 1988-1990 epidemics is significantly shorter than the clinical course of sleeping sickness experienced by the West African T.b. gambiense patients.

\section{Discussion}

The clinical course of sleeping sickness during the period from 1901 to 1910 Ugandan epidemic does not differ from contemporary T.b. rhodesiense cases in Uganda (22). The duration of illness from first onset of symptoms of documented T.b. gambiense patients did differ significantly from both the Ugandan 1901-1910 and Ugandan 1988-1990 patients. The clinical course from the

Table 2. Time sick preadmission and total time to death for 16 patients admitted to the Royal Society Sleeping Sickness Commission Hospital before 1903

\begin{tabular}{lcccc}
\hline Ref. & Patient no. & Months sick preadmission & Time in hospital (mo) & Time sick pre -death (mo) \\
\hline$(6)$ & 35 & 4 & 4.5 & 8.5 \\
$(6)$ & 23 & 1 & 2.1 & 3.1 \\
$(6)$ & 18 & 3 & 1.4 & 4.4 \\
$(6)$ & 40 & 2 & 1.2 & 3.2 \\
$(6)$ & 25 & 7 & 1.2 & 8.2 \\
$(6)$ & 22 & 2 & 1.1 & 3.1 \\
$(6)$ & 41 & 3 & 1.4 & 4.4 \\
$(6)$ & 37 & 2 & 1.9 & 3.9 \\
$(6)$ & 38 & 6 & 1.7 & 7.7 \\
$(6)$ & 15 & 1 & 1.8 & 2.8 \\
$(6)$ & 36 & 2 & 0.4 & 2.4 \\
$(6)$ & 42 & 3 & 1.9 & 4.9 \\
$(18)$ & 28 & 8 & 7.0 & 15.0 \\
$(18)$ & 20 & 6 & 3.6 & 9.6 \\
$(18)$ & 21 & 3 & 2.5 & 5.5 \\
$(18)$ & 79 & 1 & 1.6 & 2.6 \\
\hline
\end{tabular}




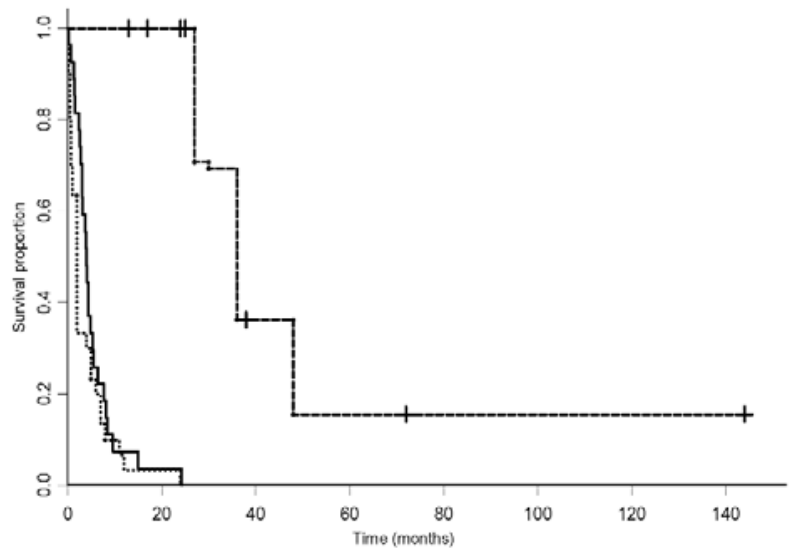

Figure 3. Results of the survival analysis (confidence intervals not shown), showing the proportion of patients surviving over the period from first onset of symptoms for the different groups of patients. The dashed line is the West African dataset from Yorke (28) (vertical bars represent censored observations). The solid line is the 1901-1910 Mengo and Sleeping Sickness Commission datasets and the dotted line the Tororo 1988-1990 dataset from Odiit et al. (22).

1901-1910 epidemic is consistent with infection with parasites belonging to the T.b. rhodesiense subspecies and is consistent with the hypothesis proposed by Köerner et al. (2). Unfortunately, no archived parasite material exists for this period, which would allow the molecular confirmation of this result by screening for the SRA gene (35), which is specific to T.b. rhodesiense, or for screening with T.b. gambiense-specific molecular markers (36).

Given these findings, how might the observations made by Castellani (16), that two distinct clinical pictures were sometimes observed in Uganda at the time, be explained? The designation as "Nubians" of some of the patients suggests that these patients were from Sudan. They were certainly migrants and may well have been carrying T.b. gambiense parasites before migrating that were discovered on examination in Uganda. Although local transmission of these parasites cannot be excluded, T.b. gambiense was probably not responsible for the widespread deaths in the Busoga region generally. Most of the cases described by Christy (7) as he roamed around Uganda were of an acute disease. Hodges, who was the Medical Officer for the Uganda Protectorate, states that the time to death of Ugandan patients from realization they were sick was 3-4 months (24), based on a great many observations, and that the duration of illness rarely exceeded 10 months. This observation is further echoed by Low and Castellani (37), who state that "very chronic cases, running a course of more than a year's duration, are very rare.” T.b. gambiense, if it did exist concurrently, would probably have been limited to those areas where migrant workers for the British authorities were allowed to settle. These settlement areas were purposefully estab- lished away from tsetse-infested bush in the efforts to control the epidemic (38).

If both species of human infective trypanosome (T.b. gambiense and T.b. rhodesiense) were in Uganda during the 1901-1910 epidemic, and both were treated at the Mengo and Sleeping Sickness Commission hospitals, the data acquired from the archives for deaths would be biased towards clinical descriptions of T.b. rhodesiense. The T.b. gambiense data presented here demonstrate that many of the patients are treated before death. For similar reasons, T.b. gambiense would be poorly represented among deaths in Mengo and the Sleeping Sickness Commission; patients would have been discharged, as the symptoms would not have been considered serious enough for them to remain in the scarce hospital beds. Also, mixed infections with both T.b. rhodesiense and T.b. gambiense could have occurred if T.b. gambiense was being transmitted locally; however, due to the acute nature of T.b. rhodesiense infections, patients infected with both parasites would most likely have been seen with T.b. rhodesiense-like symptoms.

Therefore, the results of this analysis cannot exclude T.b. gambiense as a cause of illness among some patients but can positively include T.b. rhodesiense as a cause of sleeping sickness at the time. The epidemic is likely to have been due to T.b. rhodesiense with occasional cases of T.b. gambiense in patients who had migrated from T.b. gambiense foci on the present northwestern border of Uganda (e.g., "Nubians”). Case number 136 from Mengo (Table 1) may well be one of the occasional T.b. gambiense case-patients (or the result of recall errors), as the patient reported having been ill for 24 months. This one case does not affect the outcome of the analysis, however (Figure 3).

\section{Rinderpest and Cattle Restocking}

If, as these data suggest, the 1900-1920 epidemic was due, at least in part, to T.b. rhodesiense, the question arises as to its causes, and the cause of the spread of sleeping sickness through the whole of the region to previously unaffected areas. There is no doubt that the onset of the colonial administration in Uganda resulted in social changes and population mobility (39), which had important environmental consequences (39). In referring to the causation of T.b. gambiense epidemics in central Africa, Lyons (19) blames the disruptive effects of colonization. Human movements had, however, occurred regularly throughout Africa's history (2), and the situation in Uganda was doubtless more complex.

Some other trigger, in conjunction with these factors, likely was involved in spreading sleeping sickness from the endemic foci outwards. It has been suggested that this might have been rinderpest, an infectious disease of livestock (39). The start of the sleeping sickness epidemic 
coincides with the end of the rinderpest pandemic in cattle $(39,40)$. In the early 1890 s, and for the decade that followed, rinderpest, or cattle plague, ravaged most of Africa. Millions of cattle died from this virulent viral infection (40), causing sociologic and ecologic upheavals throughout the continent. Although rinderpest is often linked to the sleeping sickness outbreaks in eastern Africa, it has been possibly linked for the wrong reasons. The diseaseinduced cattle depopulation is generally thought to have resulted in a change in the dominant vegetation in the whole region; pasture lands reverted to bush and the distribution of tsetse-fly vector of sleeping sickness expanded (41). Although these ecologic changes occurred, they may not have been directly responsible for the spread of sleeping sickness.

The movement of cattle during livestock restocking (20) may be linked to the introduction of T.b. rhodesiense sleeping sickness, a zoonosis with a principally domestic cattle reservoir, to previously unaffected areas that resulted in serious outbreaks of disease. With the large-scale local and regional movements of animals that occurred after the rinderpest pandemic, as animals were traded in the cattle-depopulated areas, trypanosomes may have moved with them. In conjunction with the expansion of tsetse distributions as the ecology changed, the setting was ripe for a major sleeping sickness problem. Ford (39) notes that in setting up the Uganda Protectorate, a great deal of cattle movements occurred, either as groups moved away from areas under British control, or in search of postrinderpest pasture. He also points out that the culture of large-scale cattle trading was well established. Local cattle movements as part of the restocking would have been extensive. T.b. rhodesiense could have spread from the core of the Busoga and other endemic foci to other tsetseinfested areas all around the northern Lake Victoria shore.

Recent molecular studies also challenge the conventional wisdom that T.b. rhodesiense spread through East Africa and to Uganda from Zambia (13), where it was first described. Tilley et al. (42) and Hide et al. (43) suggest that T.b. rhodesiense retains a stable genetic constitution through time and show that strains from Zambia are phylogenetically quite distinct from T.b. rhodesiense in Uganda. It is therefore unlikely that the parasite spread from there to Uganda. Rather, as Köerner et al. (2) suggested, T.b. rhodesiense has probably been present in southeast Uganda, either at endemic or epidemic levels (or both at different times), for hundreds of years. The dynamics of the spread of the disease, involving cattle movements and restocking (20), have probably been similar since the first association of cattle and humans in tsetse-infested areas of this part of the continent.

\section{Acknowledgments}

We thank the librarian of the Makerere Medical School at Mulago for allowing us access to the archives, Mark Woolhouse for input to discussions and advice on the manuscript, Martin Odiit and John McDermott for input to discussions, and Helma Hesse for assistance with locating T.b. gambiense patient data. We also thank the anonymous reviewers for their helpful comments.

E.M.F. was funded by the Medical Research Council (MRC), and E.M.F., P.G.C., S.C.W. and I.M. receive financial support from the Department for International Development (DFID), although the views expressed are those of the authors and not necessarily of DFID or the MRC.

Dr. Fèvre is a postdoctoral research scientist at the Centre for Tropical Veterinary Medicine, Centre for Infectious Diseases, University of Edinburgh, Scotland. His interests include field epidemiologic studies of the spatial relationships between humans, vectors, reservoirs, and environmental components of disease systems.

\section{References}

1. Langlands BW. The sleeping sickness epidemic of Uganda, 19001920: a study in historical geography. Occasional Paper No. 1. Kampala: Makerere University College, 1967.

2. Köerner T, de Raadt P, Maudlin I. The 1901 Uganda sleeping sickness epidemic revisited: a case of mistaken identity? Parasitol Today 1995;11:303-5.

3. Gibson W. More on sleeping sickness in Uganda (Letters). Parasitol Today 1996;12:40.

4. Cook JH. Notes on cases of "sleeping sickness" occurring in the Uganda Protectorate. J Trop Med 1901;4:236-9.

5. Cook JH. Sleeping sickness in Uganda. J Trop Med 1902;5:49-50.

6. Bruce D, Nabarro D. Progress report on sleeping sickness in Uganda. Reports of the Sleeping Sickness Commission of the Royal Society 1903;1:11-88.

7. Christy C. The epidemiology and etiology of sleeping sickness in Equatorial East Africa, with clinical observations. Reports of the Sleeping Sickness Commission of the Royal Society 1903;3:2-32.

8. Bruce D. Preliminary report on the tsetse fly disease or Nagana in Zululand. Durban, South Africa: Bennett and Davis;1895.

9. Forde RM. The discovery of the human Trypanosoma. Br Med J 1902;2:1741.

10. Dutton JE. Note on a Trypanosoma occurring in the blood of man. $\mathrm{Br}$ Med J 1902;2:881-4.

11. Manson P. A clinical lecture on the sleeping sickness. J Trop Med 1898;1:121-8.

12. Stephens JWW, Fantham HB. On the peculiar morphology of a trypanosome from a case of sleeping sickness and the possibility of its being a new species (T. rhodesiense). Ann Trop Med Parasitol 1910;4:343-50.

13. MacKichan IW. Rhodesian sleeping sickness in eastern Uganda. Trans R Soc Trop Med Hyg 1944;38:49-60.

14. Duke HL. Tsetse flies and trypanosomiasis: some questions suggested by the later history of the sleeping sickness epidemic in Uganda Protectorate. Parasitology 1918;11:415-29.

15. Morris KRS. The movement of sleeping sickness across Central Africa. J Trop Med Hyg 1963;66:59-76. 
16. Castellani A. Presence of Trypanosoma in sleeping sickness. Reports of the Sleeping Sickness Commission of the Royal Society 1903;1:3-10.

17. Dutton JE, Todd JL. First report of the Trypanosomiasis expedition to Senegambia. Memoirs of the Liverpool School of Tropical Medicine 1903;XI:1-57.

18. Bruce D, Nabarro D, Greig EDW. Further report on sleeping sickness in Uganda. Reports of the Sleeping Sickness Commission of the Royal Society 1903;4:2-88.

19. Lyons M. The Colonial disease: a social history of sleeping sickness in northern Zaire, 1900-1940. Cambridge: Cambridge University Press;1992.

20. Fèvre EM, Coleman PG, Odiit M, Magona JW, Welburn SC, Woolhouse MEJ. The origins of a new Trypanosoma brucei rhodesiense sleeping sickness outbreak in eastern Uganda. Lancet 2001;358:625-8.

21. Apted FIC. Clinical manifestations and diagnosis of sleeping sickness. In: Mulligan HW, Potts WH, Kershaw WE, editors. The African trypanosomiases. London: George Allen and Unwin;1970.

22. Odiit M, Kansiime F, Enyaru JCK. Duration of symptoms and case fatality of sleeping sickness caused by Trypanosoma brucei rhodesiense in Tororo, Uganda. East Afr Med J 1997;74:792-5.

23. Taelman H, Schechter PJ, Marcelis L, Sonnet J, Kazyumba G, Dasnoy $\mathrm{J}$, et al. Difluoromethylornithine, an effective new treatment of Gambian trypanosomiasis. Results in five patients. Am J Med 1987;82:607-14.

24. Hodges A. Sleeping sickness and Filaria perstans in Busoga and its neighbourhood, Uganda Protectorate. J Trop Med 1902;5:293-300.

25. Cook A. How sleeping sickness came to Uganda. East Afr Med J 1940;17:408-13.

26. Adams JH, Haller L, Boa FY, Doua F, Dago A, Konian K. Human African trypanosomiasis (T.b. gambiense): a study of 16 fatal cases of sleeping sickness with some observations on acute reactive arsenical encephalopathy. Neuropathol Appl Neurobiol 1986;12:81-94.

27. Thomas HW, Breinl A. Report on trypanosomes, trypanosomiasis and sleeping sickness. Memoirs of the Liverpool School of Tropical Medicine 1905;XVI:1-95.

28. Yorke W. Recent work on the treatment of sleeping sickness: a critical review. Trop Dis Bull 1921;18:155-74.

29. Kirchhoff LV. Use of a PCR assay for diagnosing African trypanosomiasis of the CNS: a case report. Cent Afr J Med 1998;44:134-6.

30. Petru AM, Azimi PH, Cummins SK, Sjoerdsma A. African sleeping sickness in the United States. Successful treatment with eflornithine. Am J Dis Child 1988;142:224-8.
31. Robinson B, Clark RM, King JF, Hurt B, Mohr JA. Chronic Gambian trypanosomiasis. South Med J 1980;73:516-8.

32. Otte JA, Nouwen JL, Wismans PJ, Beukers R, Vroon HJ, Stuiver PC. [African sleeping sickness in The Netherlands]. Ned Tijdschr Geneeskd 1995;139:2100-4.

33. Benhamou PH, Chandenier J, Schechter PJ, Epelbaum S, Tell GP, Haegele KD, et al. Trypanosomiase africaine de l'enfant traitée par éflornithine: un cas. Presse Med 1989;18:1199-202.

34. Parmar MKB, Machin D. Survival analysis: a practical approach. Chichester: John Wiley and Sons; 1995.

35. Welburn SC, Picozzi K, Fèvre EM, Coleman PG, Odiit M, Carrington $\mathrm{M}$, et al. Identification of human-infective trypanosomes in animal reservoir of sleeping sickness in Uganda by means of serumresistance-associated (SRA) gene. Lancet 2001;358:2017-9.

36. Jamonneau V, Garcia A, Ravel S, Cuny G, Oury B, Solano PN, et al. Genetic characterization of Trypanosoma brucei gambiense and clinical evolution of human African trypanosomiasis in Cote d'Ivoire. Trop Med Int Health 2002;7:610-21.

37. Low GC, Castellani A. Report on sleeping sickness from its clinical aspects. Reports of the Sleeping Sickness Commission of the Royal Society 1903;2:14-63.

38. Anon. Sleeping Sickness News: Uganda. Sleeping Sickness Bureau Bulletin 1909;1:314-9.

39. Ford J. The role of the Trypanosomiases in African ecology: a study of the Tsetse fly problem. Oxford: Clarendon Press; 1971.

40. Rossiter PB. Rinderpest. In: Coetzer JAW, Thomson GR, Tustin RC, editors. Infectious diseases of livestock : with special reference to southern Africa. Cape Town: Oxford University Press;1994.

41. Waller RD. Tsetse fly in Western Narok, Kenya. J Afr Hist 1990;31:81-101.

42. Tilley A, Welburn SC, Fèvre EM, Feil EJ, Hide G. Trypanosoma brucei: trypanosome strain typing using PCR analysis of Mobile Genetic Elements (MGE-PCR). Exp Parasitol 2003;104:26-32.

43. Hide G, Angus SD, Holmes PH, Maudlin I, Welburn SC. Trypanosoma brucei: Comparison of circulating strains in an endemic and an epidemic area of a sleeping sickness focus. Exp Parasitol 1998;89:21-9.

Address for correspondence: Eric M. Fèvre, Centre for Tropical Veterinary Medicine, University of Edinburgh, Easter Bush, Roslin, Midlothian, EH25 9RG, UK. fax: +44-131-445-5099, email: Eric.Fevre@ed.ac.uk

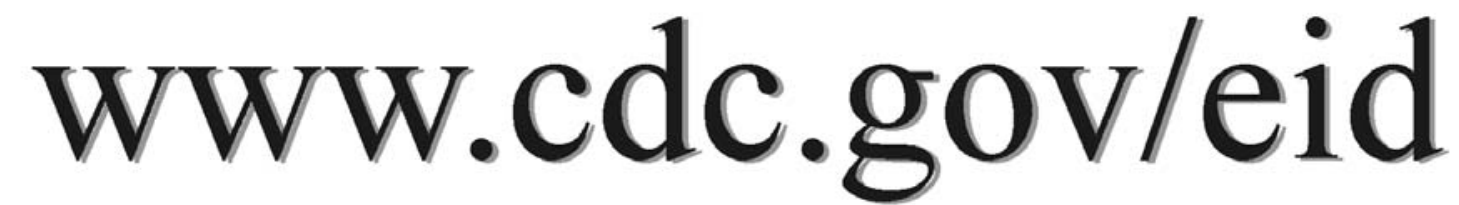

To receive tables of contents of new issues send an email to listserve@cdc.gov with subscribe eid-toc in the body of your message. 